\title{
Los estándares de cientificidad como criterio de admisibilidad de la prueba científica
}

\author{
Scientific standards as admissibility \\ requirements for scientific evidence
}

Standards de cientificidade como critérios
de admissibilidade da prova científica

Juan Manuel Alcoceba Gil ${ }^{\mathbf{1}}$

Universidad Carlos III - Madri/Espanha

jalcoceb@der-pu.uc3m.es

https://orcid.org/0000-0003-2225-0177

\begin{abstract}
Resumo: Las ciencias aplicadas son cada vez más utilizadas por la judicatura como principal vía de acceso al conocimiento de los hechos objeto de enjuiciamiento. Así lo demuestra la creciente aparición en el proceso penal de evidencias obtenidas a través de métodos de investigación de base científico-forense. Sin embargo, la legislación no contiene pautas que permitan identificar qué pruebas merecen el calificativo de científicas y cuáles no. La principal causa de esta omisión parece tener origen en la inexistencia de un concepto jurídico de ciencia, necesario para establecer los límites con que trazar la forma y contenido de la prueba científica. Solo en la jurisprudencia norteamericana pueden encontrarse ciertos criterios destinados a determinar la cientificidad de las pruebas. Estos criterios son conocidos como estándares jurisprudenciales de cientificidad
\end{abstract}

1 Doctor en Derecho por la Universidad Carlos III de Madrid. Premio extraordinario de doctorado. Desde el año 2010 imparte clases de grado y posgrado sobre diversas materias relacionadas con el proceso penal. Es coordinador de la asignatura Sistema Judicial Español y Subdirector del Master Universitario en Justicia Criminal ofertado por la Universidad Carlos III de Madrid. Ha impartido docencia tanto en universidades españolas como extranjeras y ha participado en congresos nacionales e internacionales. Es profesor del Departamento de Derecho Penal, Procesal e Historia del Derecho de la Universidad Carlos III de Madrid. 
y presentan un claro carácter heterogéneo, pues integra múltiples elementos pertenecientes a distintas nociones de ciencia. Mediante el análisis de tales estándares de cientificidad es posible apreciar el papel que cumple el Derecho en la conformación del concepto de ciencia, donde lejos de concebirse como un mero receptor pasivo, actúa como un instrumento de interacción creativa que utiliza y modifica los conocimientos científicos según sus propias exigencias.

Palabras-Chave: Proceso penal; prueba científica; jurisprudencia; estándares de cientificidad; ciencias forenses.

ABSTRACT: Applied sciences are increasingly used by the judicature as the primary means of access to knowledge of the facts under investigation or prosecution. This is evidenced by the fact that there is a growing number of evidence gathered through investigation methods that are based on forensic sciences. However, the legislation does not contain guidelines to identify which tests deserve the qualification of scientific and which do not. The main cause of this omission seems to be rooted in the inexistence of a legal concept of science, which is necessary to delimit the form and content of scientific evidence. Only in American jurisprudence can certain criteria be found to determine the scientificity of the evidence. These criteria are known as jurisprudential standards of scientificity and have a clear heterogeneous character, since they integrate elements from different notions of science. Through the analysis of such standards of scientificity it is possible to appreciate the role of law in shaping the concept of science. Thus, far from considering the law as a mere passive receptor, it may be understood as an instrument of creative interaction that uses and modifies scientific knowledge according to its own requirements.

Keywords: Criminal Procedure; Scientific Evidence; Scientific Standards; Forensic Sciences.

Resumo: As ciências aplicadas são cada vez mais utilizadas como principal via de acesso ao conhecimento dos fatos objetos do juízo. Isso é demonstrado pela crescente aparição no processo penal de provas obtidas por meio de métodos de investigação de base científico-forense. Contudo, a legislação não contém pautas que permitam identificar quais provas merecem a qualificação como científicas ou não. A principal causa dessa omissão parece ter origem na inexistência de um conceito jurídico de ciências, necessário para estabelecer os limites para traçar a forma e o conteúdo da prova científica. Somente na jurisprudência estadunidense podem ser encontrados certos critérios destinados a determinar a cientificidade das provas. Esses critérios são 
denominados "standards judiciais de cientificidade" e apresentam um claro caráter heterogêneo, pois integram vários elementos pertencentes a distintas noções de ciência. Por meio da análise de tais standards de cientificidade é possível verificar o papel que cumpre o Direito na conformação do conceito de ciência, em que, longe de se conceber como mero receptor passivo, atua como um instrumento de interação criativa que utiliza e modifica os conhecimentos científicos segundo suas próprias exigências.

Palavras-chave: Processo penal; prova científica; standards de cientificidade; ciências forenses.

SUMÁRıO: 1. El juez ante la ciencia; 2. La importancia de la prueba científica; 3. La delimitación jurisprudencial del concepto de prueba científica. Consideraciones Finales. Referencias.

\section{El Juez ante la ciencia}

Tanto la ciencia como el derecho son instituciones que han acompañado al ser humano desde los inicios de la civilización, desarrollándose junto al mismo de forma paulatina y acompasada a lo largo de la historia, lo que ha propiciado el surgimiento de espacios de interacción recíproca entre ambas disciplinas.

Si bien la esfera jurídica y científica abarcan ámbitos del conocimiento claramente diferenciados, es posible constatar como en determinadas prácticas sociales se produce una articulación conjunta de sendos paradigmas. Un claro ejemplo de ello es el ejercicio de la potestad jurisdiccional, es decir; el desempeño de la función de juzgar².

Al margen del extenso e intrincado debate mantenido por eminentes teóricos acerca del carácter científico del Derecho ${ }^{3}$, resulta patente la fuerte influencia que las ciencias naturales han ejercido sobre la

2 Para un análisis pormenorizado del paralelismo existente entre ciencia y proceso judicial, pude consultarse: ALCOCEBA GIL, Juan M. Ciencia y Proceso Penal: La prueba de ADN en el Proceso Penal Español. Tesis doctoral, Madrid, Universidad Carlos III, 2016.

3 Siendo buena prueba de la profundidad y complejidad del debate la extensísima literatura que existe en torno a dicha cuestión, de entre la que sobresale 
práctica forense a lo largo de la historia: ya sea actuando como referente epistemológico a imitar por el razonamiento judicial relativo al elemento fáctico -constitutivo de la premisa menor del silogismo jurídico clásico-4; bien sea a través del uso que se hace en el ámbito procesal de la información proporcionada por las ciencias forenses como medio de investigación y prueba ${ }^{5}$.

Así, la misma definición de prueba judicial acuñada por la doctrina más consolidada, en tanto herramienta epistémica destinada a "determinar si se pueden o no considerar verdaderos los hechos principales del caso, partiendo de que en el proceso es posible, con criterios racionales lograr una aproximación adecuada a la realidad empírica de dichos hechos”, deja entrever el peso del pensamiento científico en la conformación del actual modelo de enjuiciamiento penal ${ }^{7}$. Esta influencia resulta especialmente evidente en los sectores dogmáticos favorables a la creación de estándares de valoración racional de la prueba, pero no sólo. Las exigencias cientificistas planteadas por la academia en relación la reflexión judicial sobre los hechos, encuentran también cabida entre la jurisprudencia, siendo muestra de ello la sentencia dictada por el Tribunal Supremo Español el

la obra magna de: HART, L. A. El Concepto de Derecho. Buenos Aires: Abeledo Perrot, 1992.

4 Tesis según la cual el razonamiento efectuado por cualquiera que quiera conocer cuál es el derecho aplicable a un caso concreto (sea con fines puramente teóricos o con propósitos prácticos) se limita a la subsunción de unos hechos en unas normas jurídicas. Cuando tal ejercicio es realizado por el juez, podemos referimos a dicha teoría como teoría del silogismo judicial. ITURRALDE SESMA, Victoria. Sobre el silogismo judicial. Anuario de filosofía del derecho VIII, n. 8, p. 239-272, 1991. p. 241.

5 A través de la Criminalística; campo del conocimiento que tiene por objeto la aplicación de técnicas de investigación propias de las ciencias naturales al ámbito material de un presunto hecho delictivo con el fin de determinar, en auxilio de los órganos encargados de administrar justicia, su existencia cierta, reconstruirlo o señalar y precisar la intervención de uno o varios sujetos en el mismo (PONS, Teresa. Ciencia forense: de la teoría a la práctica. Principia, Temporada 3, Episodio 1, 2015).

6 TARUFFO, M. La prueba, Artículos y Conferencias. Buenos Aires: Metropolitana, 2008. p. 73.

7 Sobre el uso procesal de la ciencia como criterio de veracidad de las decisiones judiciales puede consultarse: WRÓBLEWSKI, J. Sentido y hecho en el derecho. México: Fontamara, 2003. p. 180 y ss. 
14 de octubre de 1986, donde el órgano judicial define el ejercicio de la jurisdicción en lo tocante al juicio histórico del siguiente modo:

"[El juez] está llamado a indagar sobre hechos y averiguar su real existencia estando para ello obligado, según se ha podido decir plásticamente, a realizar no una labor de fantasía, sino una obra de elección y construcción sobre datos preexistentes (...) no ha de ser una simple probabilidad o un mero juicio de verosimilitud, sino que ese art. 741 deba entenderse referido a que la valoración del material probatorio sea la racional y lógica o, como se denomina por relevante doctrina científica, que la prueba se aprecie con «cientificidad»".

No obstante, debe señalarse que la correlación entre pensamiento científico y reflexión judicial resulta bastante imprecisa desde una perspectiva epistemológica, así como difícilmente verificable en la práctica. Es por tanto arriesgado considerar al juez como verdadero productor de pensamiento científico ${ }^{8}$.

Donde la ciencia si encuentra de forma nítida un destacado lugar en el proceso, es al actuar como fuente de conocimiento sobre la realidad, pues en su vertiente aplicada genera información útil para la determinación de los hechos enjuiciados. Dicho de otro modo, el principal cometido del científico dentro de las salas de justicia pasa actualmente

8 Ello es así, entre otras razones debido a que: I) Frente a la amplitud de miras del científico, la libertad del juez se encuentra limitada respecto a las cuestiones que pueden ser tratadas o abordadas en el discurso de motivación. Solo dentro de los límites de la quaestio facti y de la quaestio iuris podrá el juez elaborar su discurso justificativo. Por otro lado, el producto de la actividad científica presenta vocación de universalidad, mientras que el de la actividad judicial queda necesariamente circunscrito al proceso en el que se enmarca. En este mismo sentido, las hipótesis acusatorias sobre los hechos en el proceso penal son enunciados estrictamente existenciales (no universales), que por lo tanto no pueden ser falseados (lo cual no implica que no puedan ser contradichos). En resumidas cuentas, los instrumentos de los que el sistema procesal dota al juez para la determinación de los hechos no poseen el rigor lógico-experimental sobre el que ha de sustentarse la investigación científica (ALCOCEBA GIL, J.M. Ciencia y Proceso Penal: La prueba de ADN en el Proceso Penal Español. Tesis doctoral Universidad Carlos III, 2016, p. 92 y ss). Sobre las diferencias epistemológicas existentes entre la función judicial y científica también puede consultarse: FERRUA, P. Epistemologia Scientifica ed epistemología giudiziaria: diferenze, analogie, interrelazioni. En: DE CATALLO NEUBURGER, L (org.). La prova scientifica nel processo penale. Padova: CEDAM, 2007. p. 3-28. 
por brindar información sobre lo acontecido al juez mediante la denominada prueba científica. Mediante tal mecanismo, el juzgador se sitúa frente a la ciencia en posición pasiva, recibiendo el conocimiento que esta le ofrece e integrándolo en su razonamiento probatorio. Es decir, a través de la prueba científica el juez hace suyos los postulados sobre los hechos enjuiciados que le son proporcionados por la aplicación de una determinada técnica científica al proceso. En el mismo sentido se ha pronunciado la más autorizada doctrina al enfatizar cómo "el fenómeno cada vez más relevante y frecuente del uso de pruebas científicas” viene a demostrar que "no solo no hay impermeabilidad alguna entre la determinación judicial de los hechos y el uso de metodologías científicas, sino que cada vez es más habitual que los hechos sean determinados científicamente en el proceso"9. Es por ello que cuando se analiza el rol del juez ante la ciencia, debe tenerse en cuenta que la prueba científica constituye el principal punto de unión entre ambos, al permitir que el primero asuma como propia la interpretación que la segunda le brinda sobre los hechos efectivamente acaecidos.

La prueba científica posibilita, a fin de cuentas, que "sin necesidad de que se produzca una identidad de métodos entre el juez y el científico", pueda darse uso por parte del juez de "instrumentos de análisis que permitan la valoración de los resultados producidos mediante métodos científicos"10. Por lo tanto, la denominada prueba científica constituye, a nivel epistemológico, el mecanismo mediante el cual se introduce en el proceso judicial la información arrojada por ciertas ciencias aplicadas -denominadas ciencias forenses- sobre los hechos sometidos a examen judicial, permitiendo así que el juez asuma como propia la interpretación que este campo del conocimiento le brinda sobre la realidad que debe enjuiciar en la medida que tal interpretación le sirva para fundamentar su decisión.

\section{LA IMPORTANCIA DE LA PRUEBA CIENTÍFICA}

El uso de la prueba científica se ha visto ampliamente incrementado durante las últimas décadas debido al fuerte desarrollo experimentado

9 TARUFFO, M. La prueba de los Hechos. 4a ed. Madrid: Trotta. 2011, p. 333.

10 Ibid., p. 334. 
por las ciencias forenses. Piénsese en la incidencia que ha tenido en el sistema de justicia criminal la inclusión de métodos como el análisis genético o la geolocalización de personas mediante sus terminales telefónicos. Los recientes avances en ciencia y tecnología han permitido aumentar considerablemente la eficacia del sistema de justicia criminal mediante la incorporación al proceso de nuevas y más potentes técnicas de investigación. Los descubrimientos acaecidos en materias como la física, la biología o la neurología están permitiendo a los órganos encargados de la persecución del delito recabar datos relevantes para la causa que con anterioridad resultaban imposibles de conocer ya que, en muchos casos, ni siquiera se sabía de su existencia.

Junto con el incremento en su utilización, estamos asistiendo a una intensa valorización del papel de la ciencia en el marco del proceso ${ }^{11}$. Así, la reflexión judicial de carácter fáctico que alude al juicio histórico de la sentencia, se entiende hoy mejor y más fundamentada cuando se sustenta en la existencia de pruebas científicas que la avalan ${ }^{12}$. En este contexto, métodos como la identificación por ADN no solo son cada vez más utilizados, sino que además han adquirido el valor de certeza incuestionable, llegándose a equiparar por parte de la jurisprudencia con la documentación de una realidad objetiva. De este modo, la ciencia forense representa en la actualidad la principal fuente de veracidad con la que cuentan los órganos judiciales para la determinación de los hechos.

11 En este sentido señala Vazquez-Rojas como "es común, aunque quizá cada vez menos, la idea de que aquello que se califica como científico o toda afirmación aplicable al caso hecha en el ámbito científico es particularmente eficaz para determinar con certeza los hechos del caso." (VAZQUEZ-ROJAS, C. Sobre la cientificidad de la prueba científica en el proceso judicial. Anuario de Psicología Jurídica, v. 24, n. 1, 2014, p. 66).

12 En este sentido, tal y como señala Gascón Abellán, es un sentir general el hecho de que "la prueba científica, por estar basada en leyes universales o en todo caso en leyes probabilísticas que gozan de un fuerte fundamento científico, aparece muchas veces rodeada de un áurea de infalibilidad que proporciona a la decisión probatoria un carácter concluyente o casi concluyente, por lo que no parece necesario buscar estándares adicionales que garanticen su objetividad" (GASCÓN ABELLÁN, Marina. Validez y valor de las pruebas científicas: la prueba del ADN. 2010, [en línea]. Universidad de Castilla-La Mancha [fecha de consulta: 19 de noviembre de 2017]. Disponible en: <http:// www.uv.es/CEFD/15/gascon.pdf >. Acceso en: 11 ene. 2018). 
En esta línea se expresan los resultados obtenidos por la investigación llevada a cabo en la Universidad Carlos III de Madrid bajo la dirección de la Prof. Helena Soleto Muñoz a raíz del proyecto de investigación "La convicción del tribunal en la identificación del imputado: prueba científica versus prueba testifical" cuyo objetivo ha sido comprobar la realidad empírica actual en materia de uso y consideración de la prueba científica en el proceso penal ${ }^{13}$. Según el estudio, en 275 de las 994 sentencias analizadas se realizó alguna prueba científica, esto es, en el 27,7 \% de los casos analizados. Para estos casos, la prueba que más se utilizó fue la dactiloscópica ( 59 \%), seguida de la prueba de $\operatorname{ADN}(42 \%)$.

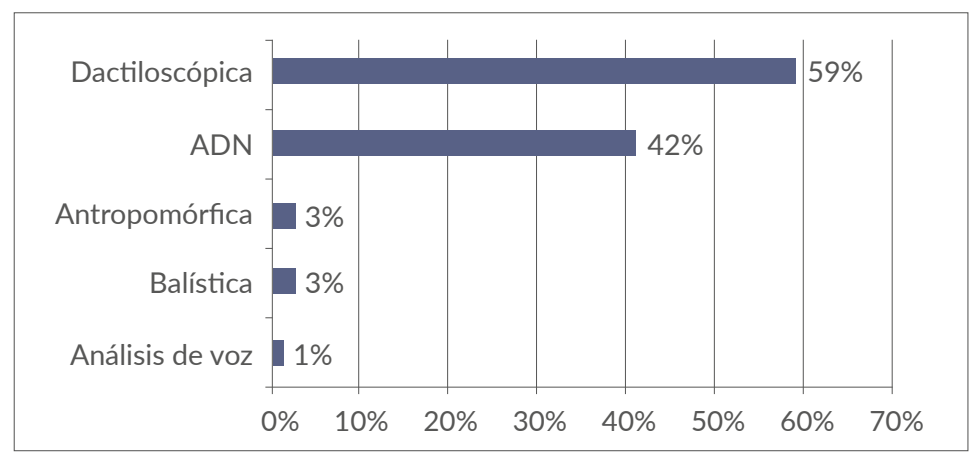

De todos aquellos casos donde se practicó la prueba de ADN, el $86,2 \%$ dio lugar a sentencias condenatorias. En los 275 casos en que se

${ }^{13}$ En el marco de este proyecto se ha realizado un amplio análisis jurisprudencial destinado a calcular el peso de la prueba científica en la convicción de los órganos judiciales penales a la hora de realizar el juicio histórico sobre los hechos. Concretamente se han clasificado las sentencias dictadas por la Audiencia Provincial de Madrid durante los años 2011, 2012, 2013 y 2014, en relación con delitos contra la vida, la integridad física, la libertad e indemnidad sexual y la salud pública. De entre la totalidad de las sentencias dictadas se seleccionaron para su categorización y posterior estudio en profundidad aquellas que recayeron sobre procedimientos donde la identidad del imputado resultaba controvertida al inicio de la investigación (994 sentencias). Ver: SOLETO MUÑOZ, H. Garantías y errores en la investigación: prueba científica versus memoria. Valencia: Tirant lo Blanch, 2015. 
utilizó alguna prueba científica, esta fue de cargo en el $92 \%$ de las veces, mientras que fue de descargo en el 7,2\% de los $\operatorname{casos}^{14}$.

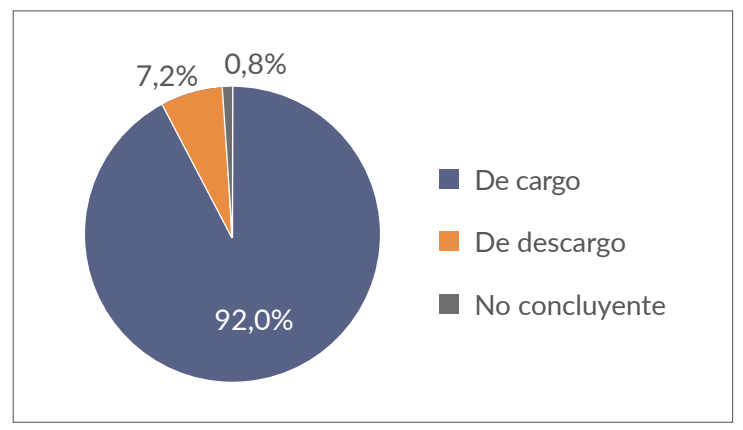

De los datos arrojados por el estudio puede inferirse que la prueba de $\mathrm{ADN}$, en tanto arquetipo de la prueba científica ${ }^{15}$, resulta casi siempre de cargo, propuesta por la acusación y dirigida a acreditar la participación del acusado en el hecho delictivo. Pero sobre todo, se desprende la alta fuerza probatoria que esta presenta. De hecho, según el mencionado estudio el índice de condenas alcanzado cuando se practica

14 Sobre la relación existente entre la prueba científica de descargo y el derecho al debido proceso se pronunció la Corte Suprema de los Estados Unidos en 1985 a través de la sentencia que pone fin al caso Ake v. Oklahoma, 470 U.S. 68. Sobre las implicaciones del pronunciamiento puede consultarse: BAILEY C., Ake v. Oklahoma and an Indigent Defendant's 'Right' to an Expert Witness: A Promise Denied or Imagined?. William \& Mary Bill of Rights Journal, v. 10, n. 2, 2002, p. 401-458.

15 Así lo indica el informe publicado en 2009 por la National Academy of Science (NAS) de Estados Unidos, bajo del título Strengthening Forensic Science in the United States. A Path Forward, según el cual el análisis genético es la única técnica forense que presenta un carácter indudablemente científico, pues no se basa en la comparación subjetiva de dos elementos de prueba, como la dactiloscopia o el examen caligráfico, sino en el cotejo de valores numéricos donde nada aporta la experiencia o parecer del perito que los realiza. COMMITTEE ON IDENTIFYING THE NEEDS OF THE FORENSIC SCIENCES COMMUNITY, NATIONAL RESEARCH COUNCIL. Strengthening Forensic Science in the United States. A Path Forward. Washington, D.C: The National Academies Press, 2009. Disponible en <http://www.nap.edu/catalog/12589. html>. Acceso en: 11 ene. 2018. 
esta prueba ante el órgano de enjuiciamiento es un $37,2 \%$ superior al obtenido cuando no se realiza ${ }^{16}$.

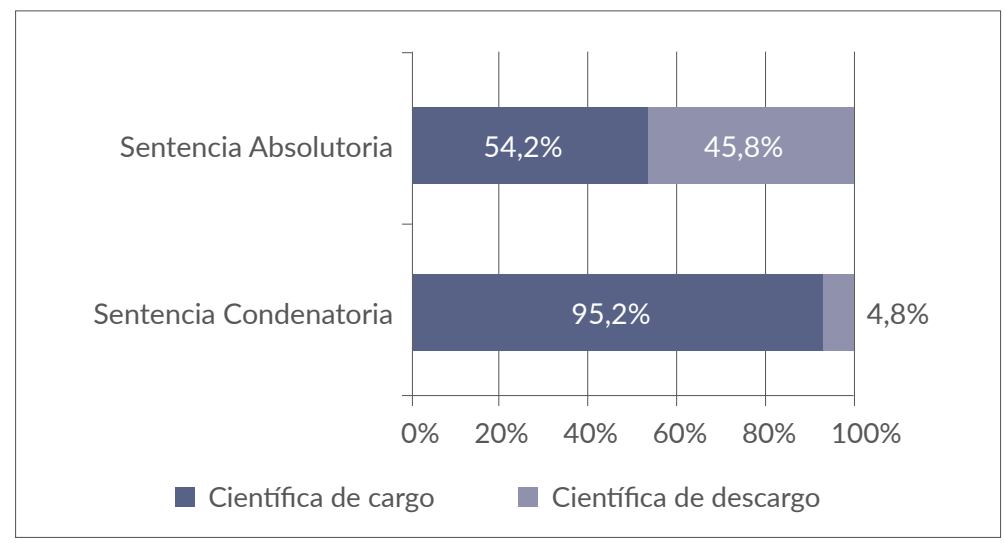

A este respecto debe advertirse que ambas características conjugadas, ser un elemento de convicción esencialmente de cargo, al que además se le atribuye una fuerza probatoria superior al resto, pueden dar lugar a la existencia de fricciones entre el uso de la prueba científica y los fundamentos acusatorios en que se inspira el modelo de proceso penal contemporáneo ${ }^{17}$. El conflicto existente entre los principios de contradicción y defensa y una concepción estatalista de la prueba científica se evidencia con mayor intensidad en sistemas como el español donde, debido su herencia inquisitorial, el principal propulsor del proceso sigue siendo la autoridad pública personificada en la figura del juez ${ }^{18}$. Es bien

16 Para mayor información sobre los resultados obtenidos a partir del citado estudio también puede consultarse: SOLETO MUÑOZ, H. Testigos y prueba científica para la identificación del acusado problemática, creencias y práctica. En: DIAZ-PICAO GIMÉNEZ, I. Derecho, justicia, universidad: liber amicorum de Andrés de la Oliva Santos. Madrid: Centro de Estudios Ramon Areces, 2016, p.3033-3054.

17 En este sentido enfoca su estudio sobre prueba pericial y adversarialidad: REDMAYNE, M. Expert Evidence and Criminal Justice. Oxford: Oxford University Press, 2011, p. 213 y ss.

18 Sobre las reminiscencias del sistema inquisitorial en el modelo mixto de enjuiciamiento penal puede consultarse: GIMENO SENDRA, J. V., MORENO 
sabido que, a diferencia de los anglosajones, en los países continentales y especialmente en España, el peso de impulsar el proceso penal una vez concurra y se mantenga la preceptiva acusación de parte recae en el juez, que es quien lo dirige con el claro objetivo de la averiguación de la $\operatorname{verdad}^{19}$. Consecuencia de ello es que en los países de tradición continental las instituciones auxiliares a la investigación presentan en la mayoría de los casos carácter público y en virtud de su oficialidad se encuentran estrechamente vinculadas a los operadores jurídicos integrantes del poder estatal $^{20}$. Bajo tal configuración del proceso, la prueba científica pasa a concebirse en la mayoría de los casos como producto de la investigación dirigida por el juez de instrucción y ejecutada por la policía judicial ${ }^{21}$, hecho que responde a la realidad práctica, pero no a la naturaleza jurídica de la misma en tanto prueba pericial ${ }^{22}$. En sistemas como el español, técnicas de investigación y prueba como el ADN o la dactiloscopia, poseen un claro carácter "oficial”, al ser introducidos siempre en el proceso por

CATENA, V y CORTÉS DOMÍNGUEZ, V. Derecho Procesal Penal. Madrid: Colex, 1999, p. 45 y ss.

19 Sobre esta cuestión puede consultarse: TARUFFO, M. Poderes probatorios de las partes y del juez en Europa” Doxa, Cuadernos de Filosofía del Derecho, n. ${ }^{\circ}$ 29, 2006, p. 249 y ss.

20 Sobre las diferencias existentes entre los sitemas de common law y civil law en relación con la producción e introducción de la prueba científica en el proceso penal, puede consultarse en general: VAN KAMPEN, P. T. C. Expert Evidence Compared: Rules and Practices in the Dutch and American Legal System. Cambridge: Intersentia, 1998.

21 Con ello no se pretende omitir la relevancia del uso de la prueba científica en el resto de las órdenes así como el hecho en que tales ordenes esta suele ser traída al proceso por las partes, sino que se expone la realidad de que en el seno del proceso penal español, francés, italiano etc. la concurrencia de la prueba científica en la fase oral o de enjuiciamiento es, en la inmensa mayoría de los casos, producto de la anterior práctica de diligencias de investigación llevadas a cabo o a instancias del juez instructor.

22 En España, la práctica totalidad de análisis genéticos que son introducidos como prueba son realizados por instituciones oficiales como: i) el Instituto Nacional de Toxicología y Ciencias Forenses, ii) los laboratorios de criminalística pertenecientes al Cuerpo Nacional de Policía y a la Guardia Civil. A este respecto puede consultarse: ALCOCEBA GIL, J.M. Ciencia y Proceso Penal: La prueba de ADN en el Proceso Penal Español. Tesis doctoral Universidad Carlos III, 2016. 
mano de la policía científica-judicial ${ }^{23}$. Así, las reminiscencias inquisitoriales presentes en el modelo continental de proceso penal, donde la actividad investigadora se entremezcla con la jurisdiccional, junto con el alto concepto que en el continente europeo se tiene (o tenía) respecto a la fiabilidad de lo público, parecen haber favorecido el monopolio absoluto de las instituciones oficiales encargadas de la investigación penal en la producción y aportación de la prueba científica ${ }^{24}$.

Más allá de la problemática señalada, tanto la especial consideración que se le otorga a la prueba científica como su creciente uso, evidencian el destacado papel que actualmente desempeña la ciencia en la reflexión judicial. Sin embargo, la legislación vigente no contiene definición alguna que permita identificar qué pruebas merecen el calificativo de científicas y cuáles no. Tampoco en la jurisprudencia española, europea o latinoamericana puede encontrarse alusión a los criterios que deben tenerse en cuenta a la hora de determinar la cientificidad de las pruebas. Puede decirse entonces que pese a la ascendente consideración que este tipo de evidencias recibe por parte de la judicatura y de la opinión pública, su naturaleza teórica ha sido ignorada por la mayoría de operadores jurídicos, quienes hasta la fecha no han reconocido esta

23 Debe tenerse en cuenta que debido a una interpretación extensiva por parte de la jurisprudencia del artículo 20 del Real Decreto 769/1987, de 19 de junio, de Policía Judicial, -mediante el cual la Policía Científica, desde el inicio de sus actuaciones, ya sea una vez incoado el procedimiento o en la fase preprocesal, genera actos de investigación que pueden constituirse como prueba de cargo en base a su difícil o imposible reproducción, tales como la toma de vestigios o muestras (ver artículos 282 y 785 LECrim)- la distinción conceptual entre actos de investigación y prueba, en la práctica de la prueba científica, se ha visto diluido hasta el punto de llegar a confundirse los unos con los otros en la llamada prueba documental científica. Véase: SSTS 10-6-1999 (5151082 Tol) , 16-7-2001 (4976517 Tol) y 3-12-2002 (240858 Tol)

A este respecto puede consultarse DOLZ LAGO, M. J. La aportación de la policía científica al proceso penal. En: DOLZ LAGO, M. J. (dir.), FIGUEROA NAVARRO, C. (coord.). La prueba pericial científica. Madrid: Edisofer, 2012, p. 35 y ss, donde se aborda de forma extendida y minuciosa la gran aportación que históricamente la policía científica ha realizado al proceso penal a través de todas sus investigaciones, sobresaliendo el enorme incremento de su capacidad a partir de las últimas dos décadas gracias al gran desarrollo de la técnica científica, idea que confirma el papel principal de las fuerzas y cuerpos de seguridad en el ámbito de la prueba científica. 
modalidad probatoria como tipología o categoría diferenciable del resto de las pruebas, sirviéndose de ella en la práctica sin delimitar legal o jurisprudencialmente los rasgos que le son propios. La principal causa de esta omisión legislativa parece tener origen en la inexistencia de un concepto jurídico de ciencia, aparentemente necesario para establecer los límites con que trazar la forma y contenido de la prueba científica.

\section{LA DELIMITACIÓN JURISPRUDENCIAL DEL CONCEPTO DE PRUEBA CIENTÍFICA}

En lo que a la prueba científica se refiere, la única preocupación del legislador iberoamericano hasta la fecha parece ha sido la de dotar de regulación concreta -que no siempre y casi nunca suficientemente-a cada una de las nuevas modalidades probatorias generadas a partir del desarrollo de la ciencia forense, sin pronunciarse en ningún caso sobre la naturaleza común a todas ellas en tanto tipología probatoria ${ }^{25}$.

Así, el legislador español, por ejemplo, ha optado por, a partir de la regulación prexistente en materia de prueba pericial ${ }^{26}$, ir paulatinamente introduciendo en el ordenamiento los diferentes remiendos legislativos que la situación requería, siempre destinados a dar cobertura normativa, más o menos improvisada, a las nuevas prácticas policiales que los recientes medios de investigación exigen ${ }^{27}$. Tal es el caso de la modificación de los artículos 326 y 362 de la Ley de Enjuiciamiento Criminal (LECrim) llevada a cabo por la Ley Orgánica 15/2003 en relación con la recogida de huellas y vestigios biológicos por parte de la policía judicial, con el fin de proceder a su análisis genético. O cuestionable modificación del artículo 788.2 LECrim operada por la disposición adicional tercera de la

25 Así podemos encontrar regulaciones, más o menos detalladas, como la del análisis genético o el análisis de sustancias estupefacientes, pero siempre de forma fragmentaria.

Medio probatorio regulado en los arts. 456 a 485 Ley de Enjuiciamiento Criminal de 14 de septiembre de 1882.

27 Sobre la regulacion de la prueba cientifica como prueba pericial em el contexto internacional puede consultarse: BACHMAIER WINTER, Lorena. Dos modelos de prueba pericial penal en el derecho comparado: Estados Unidos de Norteamérica y Alemania. Jueces para la Democracia, n. 66, p. 118-137, nov. 2009. 
Ley Orgánica 9/2002, de 10 de diciembre ${ }^{28}$-en referencia al procedimiento abreviado- y que otorga en su segundo párrafo el carácter de prueba científica documental a los informes emitidos por laboratorios oficiales en materia de naturaleza, cantidad y pureza de sustancias estupefacientes.

Ambas modificaciones legislativas, independientemente de su contenido, son ejemplo de la fragmentariedad que ha caracterizado la política seguida por el legislador español en materia de diligencias de investigación y pruebas científicas. Esta deficiente metodología se ha pretendido corregir, aunque muy parcialmente, en el anteproyecto de LECrim de $2011^{29}$ y el borrador de Código Procesal Penal $2013^{30}$. Ambos

28 No relativa a la modificación de la LECrim., sino del Código Penal y del Código Civil en materia de sustracción de menores (BOE del 11 de diciembre de 2002). En cuanto a la forma en que la LECrim ha sido modificada por esta norma: LÓPEZ CASTILLO, M.; DÍAZ CABIALEZ, J. A. La conversión de la prueba pericial en documental, artículo 788. 2. II LECr. Jueces para la Democracia, n. ${ }^{\circ}$ 46, p. 70-71, 2003. "No se ha producido el merecido debate parlamentario que merece una innovación de este calado. Se ha tratado de una introducción sorpresiva, a través de una enmienda en el Senado, en una ley que no tiene nada que ver con la reforma del proceso penal. Ciertamente la sustracción de menores no justifica la reforma de la LECrim, la LOPJ y la LORPM, porque no tiene nada que ver ni con la prueba documental en el Proceso penal, ni con los jueces en expectativa de destino, los requisitos para acceder a la condición de magistrado, ni con la supresión de la disposición transitoria única de la LO 9/2000 de medidas urgentes para agilizar la Administración de Justicia, ni tampoco con los infractores de edades comprendidas entre los 18 y 21 años. Por ello, buscar una referencia a estas cuestiones, en la exposición de motivos de la LO 9/2002 es una evidente pérdida de tiempo". Y en cuanto a su ubicación: "La conversión de la prueba pericial en documental se anticipó inconscientemente, de suerte que su ubicación temporal ha resultado disparatada: el artículo 788 LECrim, todavía en vigor, se refiere a la asistencia letrada y a la postulación del imputado. Ni siquiera se trata de la regulación del informe pericial como acto de investigación en las diligencias previas, artículo 785.7 aún vigente y futuro artículo 778 LECrim”.

Aprobado en julio de 2011 en Consejo de Ministros y decaído por la convocatoria de elecciones generales anticipadas el 20 de noviembre de ese mismo año. Se trata del único texto íntegro alternativo a la LECrim de 1882 que ha contado con la aprobación del Consejo de Ministros. La normativa contenida en el mismo en relación con la prueba científica y sobre todo con la prueba basada en el análisis de ADN ha sido la más detallada que se ha tratado de incluir en la LECrim hasta el momento.

Realizado por la Comisión institucional creada por Acuerdo de Consejo de Ministros de 2 de marzo de 2012, para la elaboración de propuestas de textos articulados de Ley de Enjuiciamiento Criminal. El texto articulado no pasó de ser una mera propuesta presentada ante el ministerio de justicia el 25 de 
textos normativos, que fueron finalmente desechados previamente a su presentación ante las cámaras, pretendían, a partir de una cierta sistemática, dotar de una regulación concreta y cohesionada a técnicas como la prueba de ADN o el análisis de sustancias, reconociendo, por tanto, ciertas especificidades en su práctica y ratificación ante el plenario.

Ante el silencio normativo existente en la materia, será la jurisprudencia norteamericana quien afronte por primera vez la difícil tarea de dotar de significado al concepto jurídico de ciencia aplicable en el marco del proceso judicial y la actividad probatoria. Tal labor se realizó mediante la formulación de ciertos estándares destinados a servir como instrumento conceptual para identificar qué técnicas de investigación y prueba presentan una base científica y cuáles deben ser rechazadas por carecer del suficiente rigor. Debe entenderse, por tanto, que el modelo de ciencia construido por la judicatura es el producto de la delimitación en sentido negativo llevada a cabo por los estándares contenidos en ciertas resoluciones judiciales donde se examina la admisibilidad de la prueba científica.

El artífice de tales estándares ha sido concretamente el Tribunal Supremo de los Estados Unidos, quien a lo largo del siglo XX dictó cuatro importantes sentencias donde se establecían los criterios que los órganos judiciales de dicho país debían tener en cuenta a la hora de admitir el expert testimony de base científica. Estos pronunciamientos son los atenientes a: (I) el caso Frye c. Estados Unidos de $1923^{31}$, (II) el caso Daubert, et al.c. Merrell Dow Pharmaceuticals de $1993^{32}$, (III) caso Joiner v. General Electric de $1997^{33}$ y (IV) caso Kumho Tire Co, Ltd. c. Carmichael de $1999^{34}$.

febrero de 2013. Fue abandonado a su suerte previamente a ser informado ante el Consejo de Ministros, por lo que, como en el caso del Anteproyecto de 2011, nunca se llegó a iniciar su tramitación parlamentaria.

31 Sentencia FRYE c. UNITED STATES. No. 3968. Disponible en: <http://www. law.ufl.edu/_pdf/faculty/little/topic8.pdf>. Acceso en: 11 ene. 2018.

32 Sentencia DAUBERT, et al. v. MERRELL DOW PHARMACEUTICALS, INC. No 92-102. Disponible en: <https://www.law.cornell.edu/supct/html/92102.ZS.html>. Acceso en: 11 ene. 2018.

33 Sentencia GENERAL ELECTRIC CO. et al. c. JOINER et ux. No. 96-188. Disponible en: <https://www.law.cornell.edu/supct/html/96-188.ZS.html>. Acceso en: 11 ene. 2018.

34 Sentencia KUMHO TIRE CO., LTD., et al. c. CARMICHAEL et al. No. 971709 Disponible en: <https://www.law.cornell.edu/supct/html/97-1709. ZS.html>. Acceso en: 11 ene. 2018. 


\subsection{El TEST FrYE DE ACEPTABILIDAD GENERAL}

El primero de los referidos pronunciamientos tuvo lugar con motivo del enjuiciamiento de James Alphonse Frye, quien fue acusado en 1920 de homicidio y sometido durante la investigación de la causa al polígrafo. Los resultados arrojados por esta técnica de investigación confirmaron la versión del investigado, que sostenía su inocencia. Sin embargo, en primera instancia la Corte de Distrito no admitió el expert testimony de los peritos que efectuaron la prueba, pues el detector de mentira se fundaba en planteamientos que por entonces no gozaban del "reconocimiento científico suficiente entre los entendidos de fisiología y psicología"35. Tal argumentación fue confirmada por el Tribunal Supremo de los Estados Unidos en 1923 mediante la referida sentencia, que sentaría el primer precedente en materia de cientificidad de la prueba, vigente hasta la década de los noventa. De acuerdo con Frye el expert testimony requiere, para poder ser admitido en juicio, la superación de un "test de aceptación general" según el cual la disciplina aplicada por el experto debe contar con un cierto nivel de reconocimiento entre la comunidad científica de referencia. En virtud de éste "test de cientificidad", una prueba científica puede ser admitida en la medida que la teoría en que se fundamenta la técnica utilizada por el experto sea aceptada por la generalidad de operadores pertenecientes al ámbito del conocimiento al que corresponde. En este sentido, el estándar Frye llegó a ser conocido como el "test de la aceptación general”. Nótese que mediante el mismo se adopta un modelo epistemológico de ciencia basado en el consenso de los "especialistas" sobre los fundamentos de cada concreta práctica, encomendándose así a los científicos la función de establecer qué es y qué no es ciencia.

El estándar de aceptación general comenzará a resultar problemático a partir de la década de los 70, ya que, debido a la naturaleza conservadora del mismo, su aplicación dificultaba la inclusión las técnicas

35 En su redacción original: "We think the systolic blood pressure deception test has not yet gained such standing and scientific recognition among physiological and psychological authorities as would justify the courts in admitting expert testimony deduced from the discovery, development, and experiments thus far made". 
que debido a su novedad y no necesariamente falta de fiabilidad, aún no habían sido abaladas por la comunidad científica ${ }^{36}$.

\subsection{LA TRILOGÍA DAUBERT}

Como segundo y principal hito se encuentra la sentencia que resolvió el caso Daubert, et al. c. Merrell Dow Pharmaceuticals en 1993. La resolución dirime la controversia suscitada a raíz de los posibles efectos nocivos del medicamento Bendectin sobre las mujeres embarazadas. Mediante su dictado el Tribunal Supremo de los Estados Unidos de América retomaría la cuestión de la cientificidad de la prueba setenta años después del primer pronunciamiento existente sobre la materia -Frye-, para establecer ciertos criterios de carácter valorativo preconizables de la metodología mediante la cual ésta ha sido producida, de tal manera que en ausencia de ellos esta no debiera ser considerada como científica ${ }^{37}$.

Estos criterios, que en su conjunto conforman el denominado estándar Daubert, resultan ser: i) La corroborabilidad empírica y falseabilidad de la teoría científica en que se sustenta su producción; ii) La posibilidad de determinar el porcentaje de error relativo a la técnica empleada; iii) La existencia de un control ejercido por otros expertos; peer review sobre la disciplina en cuestión; iv) La existencia de consenso general de la comunidad científica acerca de la validez de los planteamientos sobre los que se asienta; v) La necesidad de que exista una conexión directa entre la prueba y los hechos de los que trata el caso concreto.

Como puede apreciarse los criterios enumerados en la citada sentencia son dispares, resultando especialmente relevantes en su conjunto,

36 APPAZOV, A. Expert Evidence and International Criminal Justice. Switzerland: Springer, 2016, p. 127.

37 Dos madres que habían tenido hijos con malformaciones congénitas y las atribuían a la ingestión durante el embarazo de Bendectin demandaron a la compañía farmacéutica que produjo el medicamento. Tanto las demandantes como la compañía farmacéutica "Merrell Dow Farmaceuticals", presentaron peritos que basaban sus opiniones en estudios epidemiológicos. Al final de todo el complejo proceso judicial el Tribunal Supremo de Estados Unidos desestimó el testimonio de los peritos de las demandantes. GUTHEIL, T G., y BURSZTAJN, H J. Attorney Abuses of Daubert Hearings: Junk Science, Junk Law, or Just Plain Obstruction? Journal of the American Academy of Psychiatry and the Law, vol. 33, n. 2, 2005. p. 150-152. 
ya que suponen el primer estándar de cientificidad jurisprudencialmente establecido. De hecho, la importancia de los criterios sobre cientificidad de la prueba establecidos en la sentencia Daubert fue tal, que dio origen a la transformación del régimen jurídico aplicable a las pruebas periciales en el ordenamiento estadounidense. Transformación articulada a través de la modificación que un año después de ser dictada se llevaría a cabo sobre la regla 702 de las Federal Rules of Evidence ${ }^{38}$. Mediante la referida modificación, sin llegar a asumirse literalmente el estándar enunciado en Daubert, se introdujo un nuevo criterio de admisibilidad de la prueba científica que restringía la práctica del expert testimony ${ }^{39}$ a los supuestos en que su aportación se pudiera "fundamentar en hechos o datos suficientes, fuera el producto de principios y métodos fiables y el experto hubiera aplicado válidamente estos principios y métodos a los hechos del caso específico" ${ }^{40}$.

Esta modificación operada en las Federal Rules of Evidence, al igual que otras muchas que han tenido lugar en ordenamientos distintos al norteamericano, es una muestra de cómo mediante la profundización llevada a cabo por el Tribunal Supremo estadounidense en el concepto jurídico de ciencia, se han podido generar pautas a partir de las cuales resulte posible evaluar la admisión de toda prueba con visos de considerarse científica ${ }^{41}$.

38 Aprobadas en 1975 para ser aplicadas como criterios de admisión de la prueba en la jurisdicción federal, para un análisis pormenorizado: MUELLER, C. B.; KIRKPATRICK, L. C. Federal Rules of Evidence. Nueva York: Marcial Pons, 2005.

39 Figura procesal que encontraría su equivalencia en las pruebas periciales científicas contempladas por nuestra LECrim en los artículos 769 a 772, 777 y 778, en la fase de instrucción del procedimiento abreviado, en los arts. 723 a 725 en el plenario del procedimiento ordinario y art. 788.2 en el procedimiento abreviado.

TARUFFO, M. La prueba científica, ob. cit. p. 283.

Consultar informe: NAS 2009, Strengthening Forensic Science in the United States. A Path Forward (Committee on identifying the Needs of the Forensic Sciences Community, National Research Council, Marzo 2009); CHAMPOD, C. y VUILLE, J. Prueba científica en Europa. Admisibilidad, apreciación e igualdad de armas. Estrasburgo: Cdpc/docs 2010/cdpc (2010) 10-f.; BERGER, M. A. The Admissibility of Expert Testimony. Em: COMMITTEE ON SCIENCE, TECHNOLOGY, AND LAW POLICY AND GLOBAL AFFAIRS. Reference Manual on Scientific Evidence. Washington D.C: The National Academy, 2011, p. 11-36 
Cuatro años después de que el Juez Blackmun enunciara los criterios que integran el estándar Daubert en relación con el expert testimony prestado en el marco del proceso penal, el Tribunal Supremo norteamericano amplió su ámbito de aplicación a todo tipo de pericias por medio de la sentencia que resuelve el caso Joiner c. General Electric. Mediante el citado pronunciamiento no se introduce modificación alguna en el estándar establecido con anterioridad por el mismo Tribunal, sino que se habilita a los órganos judiciales que conocen en vía de recurso para volver a valorar la cientificidad de las pruebas científicas admitidas en primera instancia mediante la aplicación de los criterios Daubert. Esta posibilidad encuentra su justificación en la necesidad de establecer mecanismos para evitar el abuso en la discreción del juez de instancia, siendo uno de ellos aquel que permite al tribunal jerárquicamente superior evaluar si la admisión de la prueba científica ha sido suficientemente fundamentada y dicha fundamentación es acorde con la razonabilidad, la lógica y las particularidades que el caso concreto presenta ${ }^{42}$.

La última de las sentencias que conforma la denominada "trilogía Daubert" se refiere al caso Kumho Tire Co., Ltd. c. Carmichael, en el marco del cual se vuelve a extender el ámbito de aplicación de los criterios enunciados en 1993. Esta vez respecto de todo tipo de peritaje. El caso Kumho, sirvió al Tribunal para establecer la necesidad de que toda pericia, independientemente de la calidad científica del método en que se basa, sea sometida a examen según los criterios Daubert, siempre y cuando estos resulten aplicables en función de la lógica y el sentido común. En este sentido, el referido pronunciamiento hace hincapié en que el órgano judicial debe obrar como gate keeper no solo cuando la prueba pericial es científica, sino en todo caso, valorando la admisibilidad de la misma en virtud de las características particulares que ésta y la investigación en su conjunto presentan ${ }^{43}$.

42 En este sentido la sentencia señala como: "Abuse of discretion-the standard ordinarily applicable to review of evidentiary rulings-is the proper standard by which to review a district court's decision to admit or exclude expert scientific evidence".

43 Para mayor ahondamiento en el impacto que tuvo la trilogía Daubert en el ordenamiento estadounidense y por extensión en el ámbito internacional, puede consultarse. SIMONS PINO, A. La prueba científica. Revista Eletrônica de Direito Processual - REDP, v. 18, n. 3, 2017. p. 11-12. 


\subsection{La doctrina de la Corte Suprema de Canadá}

En clara conexión con los criterios Daubert, la jurisprudencia canadiense también desarrollaría en 1994 su propio estándar de cientificidad a partir del caso R. c. Mohan ${ }^{44}$. Estándar que se vería a su vez complementado por lo dispuesto en los pronunciamientos atenientes a los casos R. c. J.-L.J. ${ }^{45}$ de 2000 y R. c. Trochym de $2007^{46}$.

Mediante la primera de las referidas sentencias, el Alto Tribunal canadiense se limitó a establecer los siguientes requisitos de cara a la admisión del expert testimony: a) Que la evidencia sea relevante, b) que exista necesidad de asistir al juzgador de los hechos, c) que no exista una regla que la excluya y d) que el testimonio o ratificación pericial sea realizado por un experto cualificado. La segunda resolución exige, adicionalmente a lo previamente expuesto, la necesidad de que la ciencia o técnica deba estar fundada en planteamientos fiables, fidedignos, lo cual implica que: a) La teoría o técnica puedan y hayan sido puestas a prueba, b) la teoría o técnica hayan sido sujetas a revisión de la comunidad científica, a través de su publicación, c) se conozca el margen de error potencial o la existencia de estándares al respecto y d) la teoría sea generalmente aceptada.

El tercero de los pronunciamientos citados, matiza que no toda ciencia o técnica debe ser motivo de escrutinio previo a su admisión, pues en algunos casos, la ciencia goza de tal aceptación y reconocimiento general que los jueces pueden validar su uso sin someterla a los estándares de referencia. No obstante, el órgano judicial recuerda como el criterio sobre la fiabilidad de una determinada ciencia o técnica puede cambiar con el transcurso del tiempo, conforme avanzan los conocimientos con que se cuenta en relación con la ciencia o técnica de que se trate ${ }^{47}$.

44 Sentencia R. v. Mohan, de 5 de mayo de 1994, 2 SCR 9, n 23063. Disponible en: <https://scc-csc.lexum.com/scc-csc/scc-csc/en/item/1131/index.do>. Acceso en: 11 ene. 2018.

45 Sentencia R. v. J.-L.J. de 9 de noviembre de 2000, 2 SCR 600, no 26830. Disponible en: <https://scc-csc.lexum.com/scc-csc/scc-csc/en/item/1815/index.do>. Acceso en: 11 ene. 2018.

46 Sentencia R. v. Trochym, de 1 de febrero de 2007, 1 SCR 239, no 30717. Disponible en: <http://scc-csc.lexum.com/scc-csc/scc-csc/en/item/2341/ index.do>. Acceso en: 11 ene. 2018.

47 En este sentido, puede consultarse GOSLINGA REMÍREZ, L. Derecho, ciencia, y tecnología en la Suprema Corte Canadiense: Estándares para la 
De entre las resoluciones citadas hasta el momento, solo en el caso Daubert, et al. c. Merrell Dow Pharmaceuticals parece establecerse un verdadero estándar de cientificidad de la prueba, pues la resolución precedente (caso Frye c. Estados Unidos) acoge sin más detalle la aceptación general del método aplicado por parte de la comunidad científica ${ }^{48}$, mientras que los dos pronunciamientos posteriores a ésta (casos Joiner $v$. General Electric y Kumho Tire Co., Ltd. c. Carmichael) introducen meras matizaciones en relación al ámbito de aplicación de los criterios enunciados en 1993 y las circunstancias concretas de cada caso. Por su parte, la jurisprudencia canadiense abraza los principales elementos presentes en la línea previamente establecida por la doctrina jurisprudencial estadounidense, sin introducir modificaciones reseñables en los mismos más allá de insistir en la necesidad de valorar la pertinencia de la prueba previamente a proceder al examen de su cientificidad.

\subsection{El informe del PCAST al Presidente de los Estados Unidos}

El hito más reciente en la conformación de estándares de cientificidad de la prueba penal lo constituye un informe elaborado por el President's Council of Advisors on Science and Technology de la Casa Blanca bajo el título Forensic Science in Criminal Courts: Ensuring Scientific Validity of Feature-Comparison Methods.

El documento, publicado en septiembre de 2016, realiza un exhaustivo análisis comparado de las diferentes tecnologías forenses actualmente aceptadas como prueba ante los tribunales penales del país $^{49}$. Sus principales objetivos son, según se indica en el propio texto, clarificar los estándares científicos de validez y fiabilidad aplicables a los

admisión de evidencia científica y técnica. Scjn.gob, 2008. Disponible en: <http://www.scjn.gob.mx/Transparencia/Lists/Becarios/Attachments/77/ Becarios_077.pdf>. Acceso en: 11 ene. 2018.

48 Lo cual, más que un estándar, constituye una delegación total del órgano judicial en la comunidad científica a la hora de establecer la cientificidad de las pruebas.

49 EXECUTIVE OFFICE OF THE PRESIDENT'S COUNCIL OF ADVISORS ON SCIENCE AND TECHNOLOGY. Report to the president, Forensic Science in Criminal Courts: Ensuring Scientific Validity of Feature-Comparison Methods. Washington, D.C: PCAST Working Group, 2016 [en linea] Disponible en: <https://www.whitehouse.gov/ostp/pcast>. Acceso en: 11 ene. 2018. 
métodos forenses utilizados en los tribunales penales, así como evaluar métodos forenses específicos para determinar si son científicamente sólidos como para ser válidos y fiables. Para la consecución de tales objetivos se establecen dos nuevos criterios que todo método forense debe satisfacer a la hora de considerar su admisión: a) Validez Fundacional y b) Validez aplicacional.

La Validez Fundacional del método se entiende conferida por la existencia de estudios sobre su corroboración empírica. El estudio debe, a su vez: demostrar que el método es repetible y reproducible; proporcionar una estimación valida de su precisión (permitir el cálculo de la ratio de error). Además de alcanzar un resultado positivo en los términos expuestos, tales estudios habrán de ser realizados por múltiples grupos de investigación bajo condiciones similares a aquellas en las que está destinado a usarse en método forense. A este respecto, debe distinguirse entre la validez fundacional de los métodos objetivos -donde la opinión u especialización del perito no influyen en el resultado de la pericia-, de los métodos subjetivos -influidos por el criterio y experiencia del operador-. Para los primeros, la validez fundacional puede establecerse en relación con la medición de su precisión, reproductibilidad y consistencia expresadas en términos numéricos y por lo tanto la valoración es estadística. En el caso de los segundos, habrá de recurrirse al método "Black box studies" o doble ciego para determinar su ratio de error. Si no fuera posible establecer la ratio de acierto y error del concreto método forense, se entiende que este debe ser inadmitido como prueba. En este sentido el PCAST entiende que sin una apropiada estimación de la precisión del método, el dictamen del experto que establece la similitud o igualdad entre dos muestras carece de valor probatorio y debe ser considerado como potencialmente prejuicioso.

En cuanto la validez aplicacional o en la aplicación, el informe establece que el perito debe haber demostrado ser capaz de aplicar fiablemente el método. Especialmente en métodos subjetivos donde el juicio humano cumple un rol central. Se entiende igualmente que la habilidad en la aplicación del método de forma fiable solo puede ser demostrada a través de exámenes empíricos que midan el acierto del perito en relación con sus respuestas correctas. Los resultados de los exámenes deben ser accesibles para el resto de la comunidad científica. 
Por último, cabe señalar que el documento también alude a la forma y contenido de los informes periciales de carácter científico, estableciendo como requisitos exigibles a los mismos que: i) las aserciones plasmadas en los informes periciales sobre la fuerza probatoria de la identificación deben ser científicamente válidas y; ii) en el informe se incluya la ratio de error $\mathrm{y}$ falsos positivos que arrojen los estudios sobre la validez fundacional del método. Todo ello con el propósito general de que el perito no vaya en ningún caso más allá en sus conclusiones de lo que permitan las evidencias empíricas y la correcta aplicación de los principios estadísticos. ${ }^{50}$

Como puede observarse, los criterios de validez enunciados por el Comité de Asesores no distan demasiado de los establecidos por la jurisprudencia, siendo especialmente coincidentes en el contenido con los dos primeros criterios definidos ya en Daubert; corroborabilidad empírica de la teoría y control en la aplicabilidad del método.

\section{Consideraciones finales}

La práctica judicial penal obliga, cada vez con más frecuencia, a recurrir a fuentes de conocimiento de carácter científico que, además de resultar necesarias para resolver todos aquellos litigios cuyo objeto se encuentra estrechamente vinculado a la tecnología y la realidad científica, son concebidas en la actualidad como merecedoras de una mayor confianza que otro tipo de fuentes de conocimiento.

En este contexto se hace imprescindible de cara a la correcta regulación del proceso penal contar si no con una definición cerrada de ciencia, sí al menos con ciertos criterios que permitan discernir entre aquellas pruebas que merecen el calificativo de científicas y aquellas que no. Es decir, las actuales circunstancias que rodean al proceso penal obligan a abordar el problema de la demarcación desde la dogmática procesal, lo que supone integrar el estudio de los límites del conocimiento científico en el seno de la teoría general de la prueba.

Sin embargo, el derecho procesal se encuentra huérfano de los instrumentos epistemológicos necesarios para desarrollar su propio concepto

50 Idem. 
de ciencia autónomamente, en el proceso de elaboración de los mencionados estándares de cientificidad de la prueba la judicatura y las asociaciones científicas se ha servido de los planteamientos enunciados por distintos modelos históricos de ciencia, dando lugar a un collage de exigencias donde se entremezclan criterios de legitimidad o justificación de muy diferente naturaleza.

De este modo, en las citadas sentencias pueden encontrarse vinculados aspectos tales como la corroborabilidad empírica con la aquiescencia de la comunidad científica, la fundamentación lógica de la teoría con su reconocimiento social o el desarrollo experimental del método con la existencia de entidades que garanticen su calidad. Por todo ello, el concepto jurídico de ciencia construido por la jurisprudencia presenta un claro carácter heterogéneo, pues integra multiplicidad de elementos pertenecientes a distintas nociones de ciencia, enunciadas por diferentes teorías desarrolladas a lo largo de la historia desde epistemologías dispares, sin asumir íntegramente los postulados de ninguna de ellas.

No obstante, lejos de ser una contra ventaja, la ambivalencia teórica que muestra el concepto jurídico de ciencia resulta especialmente acertada si se tiene en cuenta el carácter irresoluble del problema al que pretende hacer frente. Ante un paradigma de pensamiento en constante cambio, cuyos límites resultan difusos incluso para aquellos campos del pensamiento centrados en su estudio, los tribunales han dejado de ser una institución epistemologicamente pasiva. La creación y evolución de los estándares de cientificidad pone de manifiesto como el Derecho no solo recibe de la ciencia una interpretación sobre los hechos, sino que también participa activamente en el debate de la demarcación, exponiendo sus propios planteamientos sobre lo que es y no es conocimiento científico y expresando sus propias exigencias sobre las teorías y técnicas que pretenden operar como pruebas. No obstante, dada la complejidad de la cuestión parece acertado que, en este marco, el Derecho adopte una perspectiva lo más multidisciplinar posible, actualizando sus planteamientos a medida que las tendencias científicas y filosóficas cambian.

\section{REFERENCIAS}

ALCOCEBA GIL, Juan M. Ciencia y Proceso Penal: La prueba de ADN en el Proceso Penal Español. Tesis doctoral, Madrid, Universidad Carlos III, 2016. 
APPAZOV, A. Expert Evidence and International Criminal Justice. Switzerland: Springer, 2016.

BACHMAIER WINTER, L. Dos modelos de prueba pericial penal en el derecho comparado: Estados Unidos de Norteamérica y Alemania. Jueces para la Democracia, n. 66, p. 118-137, 2009.

BAILEY C., Ake v. Oklahoma and an Indigent Defendant's 'Right' to an Expert Witness: A Promise Denied or Imagined?, William \& Mary Bill of Rights Journal. v. 10 , n. 2 , p. $401-458,2002$

BERGER, M. A. The Admissibility of Expert Testimony. Em: COMMITTEE ON SCIENCE, TECHNOLOGY, AND LAW POLICY AND GLOBAL AFFAIRS. Reference Manual on Scientific Evidence. Washington D.C: The National Academy, 2011, p. 11-36

COMMITTEE ON IDENTIFYING THE NEEDS OF THE FORENSIC SCIENCES COMMUNITY, NATIONAL RESEARCH COUNCIL. Strengthening Forensic Science in the United States. A path Forward. Washington, D.C: The National Academies Press, 2009. Disponible en: <http://www.nap.edu/catalog/12589.html>. Acceso en: 11 ene. 2018.

DOLZ LAGO, M. J. La aportación de la policía científica al proceso penal. En: DOLZ LAGO, M. J. (dir.). FIGUEROA NAVARRO, C. (coord.). La prueba pericial científica. Madrid: Edisofer, 2012.

EXECUTIVE OFFICE OF THE PRESIDENT PRESIDENT'S COUNCIL OF ADVISORS ON SCIENCE AND TECHNOLOGY. REPORT TO THE PRESIDENT Forensic Science in Criminal Courts: Ensuring Scientific Validity of Feature-Comparison Methods. Washington, D.C: PCAST Working Group, 2016 [en linea] Disponible en <https://www.whitehouse.gov/ostp/pcast>. Acceso en: 11 ene. 2018.

FERRUA, P. Epistemologia Scientifica ed epistemología giudiziaria: diferenze, analogie, interrelazioni. En: DE CATALLO NEUBURGER, L (org.). La prova scientifica nel processo penale. Padova: CEDAM, 2007.

GASCÓN ABELLÁN, Marina. Validez y valor de las pruebas científicas: la prueba del ADN. 2010, [en línea]. Universidad de Castilla-La Mancha [fecha de consulta: 19 de noviembre de 2017]. Disponible en: <http://www.uv.es/CEFD/15/gascon. pdf>. Acceso en: 11 ene. 2018.

GIMENO SENDRA, J. V., MORENO CATENA, V y CORTÉS DOMÍNGUEZ, V. Derecho Procesal Penal. Madrid: Colex, 1999.

GÓMEZ SÁNCHEZ, Yolanda. La protección de los datos genéticos: El derecho a la autodeterminación informativa. Derecho y Salud, vol. 16 (Extra 1), p. 59-78, 2008. 
GOSLINGA REMÍREZ, Lorena. Derecho, ciencia, y tecnología en la Suprema Corte Canadiense: Estándares para la admisión de evidencia científica y técnica. 2008. Disponible en: <http://www.scjn.gob.mx/Transparencia/Lists/Becarios/ Attachments/77/Becarios_077.pdf>. Acceso en: 11 ene. 2018.

GUTHEIL, T G., y BURSZTAJN, H J. Attorney Abuses of Daubert Hearings: Junk Science, Junk Law, or Just Plain Obstruction? Journal of the American Academy of Psychiatry and the Law, vol. 33, n. 2, 2005.

HART, Herbert Lionel A. El Concepto de Derecho, 2a edición, Buenos Aires: Abeledo Perrot, 1968.

LÓPEZ CASTILLO, M.; DÍAZ CABIALEZ, J. A. La conversión de la prueba pericial en documental, artículo 788. 2. II LECr. Jueces para la Democracia, n. ${ }^{\circ}$ 46, p. 70-71, 2003.

MUELLER, C. B.; KIRKPATRICK, L. C. Federal Rules of Evidence. Nueva York: Marcial Pons, 2005.

ITURRALDE SESMA, Victoria. Sobre el silogismo judicial. Anuario de filosofía del derecho VIII, n. 8, p. 239-272, 1991.

PONS, Teresa. Ciencia forense: de la teoría a la práctica. Principia, Temporada 3, Episodio 1, 2015.

SIMONS PINO, A. La prueba científica. Revista Eletrônica de Direito Processual - REDP, v. 18, n. 3, p. 04-44, 2017. https://doi.org/10.12957/redp.2017.31690

SOLETO MUÑOZ, H. Garantías y errores en la investigación: prueba científica versus memoria. Valencia: Tirant lo Blanch, 2016.

SOLETO MUÑOZ, H. Testigos y prueba científica para la identificación del acusado: problemática, creencias y práctica”. En: DIAZ-PICAO GIMÉNEZ, I. Derecho, justicia, universidad: liber amicorum de Andrés de la Oliva Santos. Madrid: Centro de Estudios Ramon Areces, 2016, p. 3033-3054.

TARUFFO, M. La prueba. Artículos y Conferencias, $1^{\text {a }}$ edición, Buenos Aires: Metropolitana, 2009.

TARUFFO, M. La prueba de los Hechos, 4ª edición. Madrid: Trotta, 2011.

TARUFFO, M. Poderes probatorios de las partes y del juez en Europa. Doxa, Cuadernos de Filosofía del Derecho, n. 29, p. 249-271, 2006. https://doi.org/10.14198/ doxa2006.29.13 
VAN KAMPEN, P. T. C. Expert Evidence Compared: Rules and Practices in the Dutch and American Legal System. Cambridge: Intersentia, 1998.

VAZQUEZ-ROJAS, C. Sobre la cientificidad de la prueba científica en el proceso judicial. Anuario de Psicología Jurídica, v. 24, n. 1, p. 65-73, 2014. https://doi. org/10.1016/j.apj.2014.09.001

WRÓBLEWSKI, J. Sentido y hecho en el derecho. México: Fontamara, 2003.

\section{Informações adicionais e declarações dos autores (integridade científica)}

Declaração de conflito de interesses (conflict of interest declaration): o autor confirma que não há conflitos de interesse na realização das pesquisas expostas e na redação deste artigo.

Declaração de autoria e especificação das contribuições (declaration of authorship): todas e somente as pessoas que atendem os requisitos de autoria deste artigo estão listadas como autores; todos os coautores se responsabilizam integralmente por este trabalho em sua totalidade.

Declaração de ineditismo e originalidade (declaration of originality): o autor assegura que o texto aqui publicado não foi divulgado anteriormente em outro meio e que futura republicação somente se realizará com a indicação expressa da referência desta publicação original; também atesta que não há plágio de terceiros ou autoplágio. 
Dados do processo editorial

(http://www.ibraspp.com.br/revista/index.php/RBDPP/about/editorialPolicies)

- Recebido em: 10/12/2017

Equipe editorial envolvida

- Controle preliminar e verificação de plágio:

- Editor-chefe: 1 (VGV) 19/12/2017

- Avaliação 1: 28/12/2017

- Avaliação 2: 10/01/2018

- Editor-associado: 1 (GHB)

- Revisores: 2

- Decisão editorial preliminar: 11/01/2018

- Retorno rodada de correções: 02/02/2018

- Decisão editorial final: 03/02/2018

\section{COMO CITAR ESTE ARTIGO:}

ALCOCEBA GIL, Juan Manuel. Los estándares de cientificidad como criterio de admisibilidad de la prueba científica. Revista Brasileira de Direito Processual Penal, Porto Alegre, vol. 4, n. 1, p. 215-242, jan./abr. 2018.

https://doi.org/10.22197/rbdpp.v4i1.120

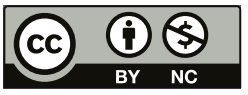

Esta obra está licenciada com uma Licença Creative Commons Atribuição-NãoComercial 4.0 Internacional. 